\title{
Killer cell immunoglobulin-like receptor 3DL1 variation modifies HLA-B*57 protection against HIV-1
}

\author{
Maureen P. Martin, ${ }^{1}$ Vivek Naranbhai, ${ }^{2,3}$ Patrick R. Shea, ${ }^{4}$ Ying Qi, ${ }^{1}$ Veron Ramsuran, ${ }^{1,3,5}$ Nicolas Vince, ${ }^{1,6,7}$ Xiaojiang Gao, ${ }^{1}$ \\ Rasmi Thomas, ${ }^{8,9}$ Zabrina L. Brumme, ${ }^{10,11}$ Jonathan M. Carlson, ${ }^{12}$ Steven M. Wolinsky, ${ }^{13}$ James J. Goedert, ${ }^{14}$ Bruce D. Walker, ${ }^{2}$ \\ Florencia P. Segal, ${ }^{15}$ Steven C. Deeks, ${ }^{16}$ David W. Haas, ${ }^{17}$ Stephen A. Migueles, ${ }^{18}$ Mark Connors, ${ }^{18}$ Nelson Michael, ${ }^{8,9}$ \\ Jacques Fellay, ${ }^{19}$ Emma Gostick, ${ }^{20,21}$ Sian Llewellyn-Lacey, ${ }^{20,21}$ David A. Price, ${ }^{20,22}$ Bernard A. Lafont, ${ }^{23}$ Phillip Pymm, ${ }^{24}$ \\ Philippa M. Saunders, ${ }^{25}$ Jacqueline Widjaja, ${ }^{25}$ Shu Cheng Wong, ${ }^{25}$ Julian P. Vivian, ${ }^{24}$ Jamie Rossjohn, ${ }^{20,21,24}$ \\ Andrew G. Brooks, ${ }^{25}$ and Mary Carrington ${ }^{1,2}$
}

'Cancer and Inflammation Program, Leidos Biomedical Research Inc., Frederick National Laboratory for Cancer Research, Frederick, Maryland, USA. Ragon Institute of MCH, MIT and Harvard, Boston, Massachusetts, USA. ${ }^{3}$ Centre for the AIDS Programme of Research in South Africa (CAPRISA), University of KwaZulu-Natal, Durban, South Africa. ${ }^{4}$ Institute for Cenomic Medicine, Columbia University, New York, New York, USA. ${ }^{5}$ KwaZulu-Natal Research Innovation and Sequencing Platform (KRISP), School of Laboratory Medicine and Medical Sciences, University of KwaZulu-Natal, Durban, South Africa. ${ }^{6}$ ATIP-Avenir, Centre de Recherche en Transplantation et Immunologie, UMR 1064, INSERM, Université de Nantes, Nantes, France. Institut de Transplantation Urologie Néphrologie (ITUN), Centre Hospitalier Universitaire (CHU) de Nantes, Nantes, France. ${ }^{8}$ US Military HIV Research Program, Walter Reed Army Institute of Research, Silver Spring, Maryland, USA. ${ }^{9}$ Henry M. Jackson Foundation for the Advancement of Military Medicine, Bethesda, Maryland, USA. ${ }^{10}$ Faculty of Health Sciences, Simon Fraser University, Burnaby, British Columbia, Canada. "British Columbia Centre for Excellence in HIV/AIDS, Vancouver, British Columbia, Canada. ${ }^{12}$ Microsoft Research, Redmond, Washington, USA. ${ }^{13}$ Division of Infectious Diseases, Northwestern University Feinberg School of Medicine, Chicago, Illinois, USA. ${ }^{14}$ Infections and Immunoepidemiology Branch, Division of Cancer Epidemiology and Genetics, National Cancer Institute (NCI), NIH, Bethesda, Maryland, USA. ${ }^{15 B r i g h a m ~ a n d ~ W o m e n ' s ~ H o s p i t a l, ~ B o s t o n, ~ M a s s a c h u s e t t s, ~ U S A . ~}$ ${ }^{16}$ San Francisco General Hospital Medical Center, San Francisco, California, USA. ${ }^{17}$ Vanderbilt University School of Medicine, Nashville, Tennessee, USA. ${ }^{18}$ Laboratory of Immunoregulation, National Institute of Allergy and Infectious Diseases (NIAID), NIH, Bethesda, Maryland, USA. ${ }^{19}$ School of Life Sciences, Swiss Federal Institute of Technology, Lausanne, Switzerland. ${ }^{20}$ Cardiff University School of Medicine, Heath Park, University Hospital of Wales, Cardiff, United Kingdom. ${ }^{21}$ Non-Human Primate Immunogenetics and Cellular Immunology Unit, NIAID, NIH, Bethesda, Maryland, USA. ${ }^{22}$ Human Immunology Section, Vaccine Research Center, National Institute of Allergy and Infectious Diseases, NIH, Bethesda, Maryland, USA. ${ }^{23}$ Viral Immunology Section, Office of the Scientific Director, NIAID, NIH, Bethesda, Maryland, USA. ${ }^{24}$ Infection and Immunity Program and Department of Biochemistry and Molecular Biology, Biomedicine Discovery Institute, and Australian Research Council Centre of Excellence in Advanced Molecular Imaging, Monash University, Clayton, Victoria, Australia. ${ }^{25}$ Department of Microbiology and Immunology, Peter Doherty Institute for Infection and Immunity, The University of Melbourne, Parkville, Victoria, Australia.

\begin{abstract}
HLA-B* 57 control of HIV involves enhanced $C D 8^{+} T$ cell responses against infected cells, but extensive heterogeneity exists in the level of HIV control among $B^{*} 57^{+}$individuals. Using whole-genome sequencing of untreated $B^{*} 57^{+}$HIV-1-infected controllers and noncontrollers, we identified a single variant (rs643347A/C) encoding an isoleucine-to-valine substitution at position 47 (I47V) of the inhibitory killer cell immunoglobulin-like receptor KIR3DL1 as the only significant modifier of $B * 57$ protection. The association was replicated in an independent cohort and across multiple outcomes. The modifying effect of $147 \mathrm{~V}$ was confined to $B * 57: 01$ and was not observed for the closely related $B * 57: 03$. Positions 2,47 , and 54 tracked one another nearly perfectly, and 2 KIR3DL1 allotypes differing only at these 3 positions showed significant differences in binding $B^{*}$ 57:01 tetramers, whereas the protective allotype showed lower binding. Thus, variation in an immune NK cell receptor that binds $B^{*}$ 57:01 modifies its protection. These data highlight the exquisite specificity of KIR-HLA interactions in human health and disease.
\end{abstract}

\section{Introduction}

HIV-1 disease progression is influenced by host genetic factors and varies greatly among infected individuals. Polymorphism in the HLA class I locus has been consistently shown to associate with outcome of untreated HIV-1 infection by both the candidate gene approach (1) and GWAS $(2,3)$. The influence of $H L A-B$ alleles is particularly robust, among which $H L A-B^{\star} 57$ and $-B^{\star} 27$ show

Authorship note: MPM and VN contributed equally to this work. Conflict of interest: The authors have declared that no conflict of interest exists. Submitted: November 6, 2017; Accepted: February 13, 2018. Reference information: J Clin Invest. 2018;128(5):1903-1912. https://doi.org/10.1172/JCI98463. consistent protective effects (4-8), and $H L A-B^{\star} 35$ subtypes are associated with accelerated disease progression (9). $B^{\star} 57$ is highly enriched in rare individuals who maintain undetectable viral loads (VLs) (plasma HIV-1 RNA $<50$ copies/ml) in the absence of antiretroviral therapy (10). Up to $50 \%$ of these "elite controllers" carry $B^{\star} 57$ alleles as compared with a frequency of $7 \%$ to $8 \%$ in noncontrollers (11) or the general white population. Notably, however, in the absence of antiretroviral therapy, most $B^{\star} 57^{+}$HIV-infected individuals fail to control HIV and typically progress to AIDS with a temporal range similar to that of individuals without $B * 57$ (8), indicating the presence of distinct modifiers of $B^{\star} 57$ protection.

The mechanistic role of $H L A-B^{\star} 57$ in controlling the virus is not fully understood, but data suggest that immune respons- 
Table 1. Allele frequency of $r$ s643347G/A in 2 independent $B * 57^{+}$cohorts $^{A}$

\begin{tabular}{lcccccc}
\multicolumn{3}{c}{ WCS, $\boldsymbol{n}=159^{\mathrm{B}}$} & \multicolumn{3}{c}{ Validation, $\boldsymbol{n}=\mathbf{5 1 0}$} \\
rs643347 allele & CTR, $\boldsymbol{n}(\%)$ & NC, $\boldsymbol{n}(\%)$ & $\boldsymbol{P}$ value & CTR, $\boldsymbol{n}(\%)$ & NC, $\boldsymbol{n}(\%)$ & $\boldsymbol{P}$ value \\
$\mathrm{G}$ & $73(43.5)$ & $25(16.7)$ & $2.4 \times 10^{-7}$ & $338(56.9)$ & $203(47.7)$ & 0.004 \\
$A$ & $95(56.5)$ & $125(83.3)$ & & $256(43.1)$ & $223(52.3)$ &
\end{tabular}

${ }^{A}$ Single-variant analysis using Fisher's exact test. ${ }^{B}$ High-quality sequence data for $147 \mathrm{~V}$ were available for 159 of the 187 samples that were included in the genome-wide analysis. predicted functional consequences (e.g., nonsynonymous, splice site acceptor/ donor, start/stop loss or gain, frameshift variants). Only a single variant, rs643347A/G, located in the KIR3DL1 gene, remained statistically significant after Bonferroni correction for multiple testing (Supplemental Figure 1). This es mediated through both acquired and innate mechanisms are involved. $\mathrm{B}^{\star} 57$ binds several immunodominant epitopes located in conserved regions of Gag, implicating $\mathrm{B}^{*} 57$-restricted $\mathrm{CD}^{+} \mathrm{T}$ cell responses in controlling viral replication (12-14). Escape mutations within these $B^{\star} 57$-restricted epitopes can result in reduced viral fitness (15-19). Viral adaptation to host HLA genotypes results in escape from cytotoxic T lymphocyte (CTL) responses, and high levels of adaptation appear to have profound deleterious effects on viral control, even among $B^{\star} 57^{+}$individuals (20). Reduced viral fitness does not compensate for the loss of an effective CTL response, and HIV controllers with $B^{\star} 57$ have significantly lower adaptation scores than do $B^{\star} 57$ noncontrollers. Nevertheless, CTLs from patients with $B^{\star} 57$ are more cross-reactive to various HIV epitopes after point mutations in these epitopes have occurred relative to CTLs from patients with $B^{\star} 08$, an allele that is associated with more rapid disease progression (21). This observation is supported by computational modeling of thymic selection, in which a larger fraction of the naive repertoire of $\mathrm{B}^{\star} 57$-restricted $\mathrm{T}$ cell clones is specific for HIV relative to that of other alleles, and $\mathrm{B}^{\star} 57$-restricted $\mathrm{T}$ cells are more cross-reactive to mutants of targeted epitopes (22), possibly impeding viral adaptation in $B^{\star} 57^{+}$individuals.

Here, we identify a nonsynonymous variant in the killer cell immunoglobulin-like receptor (KIR3DL1) gene (I47V) that modifies $B^{\star} 57$ protection against HIV- 1 . To our knowledge, this is the only locus identified genome-wide that associates with the level of $B^{\star} 57$ control, and the effect was replicated in an independent cohort of $B^{\star} 57^{+}$HIV-1-infected subjects. The protective effect of I47V was restricted to the $B^{\star} 57: 01$ subtype, and no such effect was observed for the closely related $B^{\star} 57: 03$. These 2 allotypes differ only at positions 114 and 116, located in the $\mathrm{E}$ and $\mathrm{F}$ pockets, respectively, which specify peptide binding, suggesting that differences in the positioning or sequence of peptides bound to $\mathrm{B}^{\star} 57$ are in part responsible for the protective effect of KIR3DL1 47V.

\section{Results}

The KIR3DL1 rs643347G variant is enriched in $B^{*} 57^{+}$HIV-1-infected controllers. We performed whole-genome sequencing (WGS) of $B^{\star} 57^{+}$controllers $(n=100)$ and $B^{\star} 57^{+}$noncontrollers $(n=100)$ (Supplemental Table 1; supplemental material available online with this article; https://doi.org/10.1172/JCI98463DS1) in order to identify host genetic modifiers of $B^{\star} 57$ control of HIV-1. The controller group used in the whole-genome study was highly enriched for elite controllers ( $89 \%$ with a VL $<500$ copies $/ \mathrm{ml}$ ). Sequence data were of high quality for 187 samples, including 97 controllers and 90 noncontrollers, allowing for statistical analysis of 56,808 variants genome wide that were chosen on the basis of variant results in an isoleucine ( $r s 643347 A)$ to valine ( $r s 643347 G)$ aa substitution at codon 47 within the Do domain of the mature KIR3DL1 protein, and $r s 643347 G$ was associated with elite control $\left(P=2.4 \times 10^{-7}\right.$, Fisher's exact test; Table 1$)$. Two additional variants within the KIR genomic region, $r s 1049150$ (isoleucine to leucine at aa 54 in KIR3DL1) and rs1049215 (threonine to alanine at aa 115 in KIR2DL4), both of which are in linkage disequilibrium (LD) with rs643347 $\left(r^{2}=0.93, \mathrm{D}^{\prime}=1\right.$, and $r^{2}=0.76, \mathrm{D}^{\prime}=0.99$, respectively), were also identified, but these did not remain statistically significant after correction (Supplemental Figure 1).

In order to validate the WGS results, we analyzed data from an independent $B^{\star} 57^{+}$cohort consisting of $297 \mathrm{HIV}^{+}$individuals with a more lenient definition of control ( $\mathrm{VL}<2,000$ viral RNA copies/ml of plasma) and 213 noncontrollers (VL >10,000 viral RNA copies/ml of plasma) (Supplemental Table 1 ). This cohort had a broader range of clinical phenotypes relative to the extreme phenotypes of the initial cohort in which WGS was performed, particularly with respect to the controllers (89\% elite controllers in the WGS cohort vs. $<60 \%$ in the validation cohort, in which an elite controller is defined as having undetectable VLs using standard assays). KIR3DL1 subtyping was performed in the validation cohort and confirmed the association of $47 \mathrm{~V}$ with significantly better HIV control (56.9\% in controllers vs. $47.7 \%$ in noncontrollers; $P=0.004$; Table 1). The weaker effect we observed in the validation cohort was consistent with the broader range of clinical phenotypes in this cohort relative to the discovery cohort.

Variants encoding aa 2, 47, and 54 have the strongest effects on $H I V-1$ control relative to other KIR3DL1 nonsynonymous variants. KIR3DL1 is a highly polymorphic locus that contains many nonsynonymous variants (http://www.ebi.ac.uk/ipd/kir/) that are in strong LD with one another. While WGS pointed to aa 47 as having the greatest effect on $B^{\star} 57$ control of HIV-1, other variants within the gene that may not have reached genome-wide significance because of poor detection quality could contribute to the effect observed with aa 47 . We therefore used the pooled data from the WGS and validation cohorts to test for effects of each nonsynonymous variant within the KIR3DL1 gene. Homozygotes plus heterozygotes of the lower-frequency allele were compared with homozygotes of the higher-frequency allele in order to gain power when testing rarer allelic variants. Variants encoding aa positions 2, 47, and 54 are in strong LD (ref. 23 and Supplemental Table 2), and thus the effect of each of these 3 aa was comparable (odds ratio $[\mathrm{OR}]=0.2-0.3, P=0.003-0.0003)$. We observed that aa $283 \mathrm{had}$ a weak effect $(\mathrm{OR}=0.6, P=0.02)$, but no other aa variant in the KIR3DL1 molecule significantly modified the effect of $B^{*} 57$ on HIV control (data not shown). Thus, I47V tags 2 additional coding vari- 


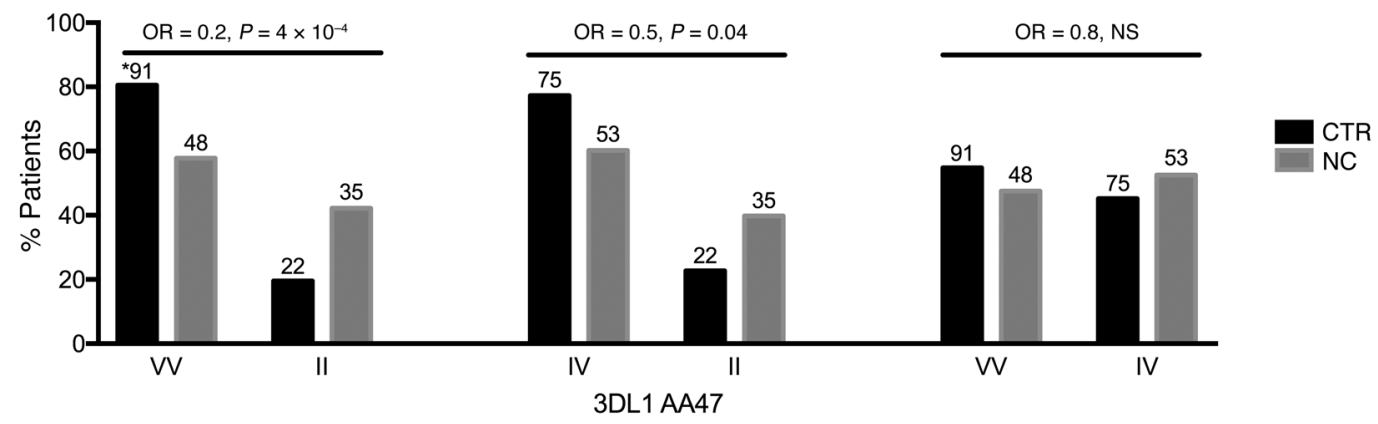

Figure 1. Influence of KIR3DL1 aa 47 on HIV control in HLA-B*57+ individuals. Only individuals with 2 expressed KIR3DL1 alleles were included in the analysis. Individuals with KIR3DS1, KIR3DL1*004 (1 or 2 copies), and KIR3DL1+/- (i.e., KIR3DL1/S1 missing on 1 haplotype) were excluded. Statistical analysis was performed using logistic regression. CTR, HIV controllers ( $n=188)$; NC, noncontrollers $(n=136)$. The numbers above the bars denote the number of individuals in each group.

ants, and although I47V appears to be the most statistically robust, any 1 or combination of the 3 aa variants at positions 2,47 , and 54 may be responsible for modulating $B^{\star} 57$ protection against HIV-1.

Genotypes encoding KIR3DL1 aa 47 (I47V) act in a codominant fashion to impact HLA-B ${ }^{*} 57$ control of $H I V-1$. Our initial analyses of I47V considered allelic (rather than genotypic) frequencies of $47 \mathrm{I}$ versus $47 \mathrm{~V}$ (Table 1), and samples carrying only 1 functional copy of KIR3DL1 (i.e., those samples in which the other haplotype carried either the activating KIR3DS1 or KIR3DL1*004, an allele that is not expressed on the cell surface) or no copies of functional KIR3DL1 were included in the analyses. KIR3DL1 allele frequencies are shown in Supplemental Figure 2 and Supplemental Table 3. As KIR3DL1 ${ }^{*} 004$ does not bind $\mathrm{B}^{\star} 57$ expressed on target cells $(24,25)$, we reasoned that this allele would be functionally irrelevant in the analyses performed herein. Furthermore, both KIR3DS1 and KIR3DL1*004 may actually confound our results, since both have been associated with protection in HIV cohorts previously (albeit not in combination with $B^{*} 57$ specifically) (26, $27)$. In order to eliminate any potential confounding effect of KIR3DS1 and KIR3DL1*004 on the modulation of $B^{\star} 57$ protection against HIV, we excluded subjects with these 2 alleles and limited the genotypic analyses to subjects with 2 copies of KIR $3 D L 1$ (i.e., those with only a single copy or $>2$ copies of KIR $3 D L 1$ were excluded), since variable gene copy numbers might also influence our analysis (28). We then tested the 3 strict genotypes of I47V (i.e., $\mathrm{VV}, \mathrm{IV}$, and II) for their frequencies among controllers (mean VL [mVL] $<2,000$ viral RNA copies/ml plasma) as compared with frequencies in noncontrollers ( $\mathrm{mVL}>10,000$ viral RNA copies/ ml plasma), all of whom were $B^{\star} 57^{+}$(Supplemental Table 1). The $47 \mathrm{VV}$ genotype was strongly associated with protection relative to 47II (OR $=0.2, P=4 \times 10^{-4}$; Figure 1 and Supplemental Table 4), and the effect appeared to be primarily codominant $(P$ for trend $=$ 0.001 ), as 47IV tended to be less protective than 47VV (though not significantly) and more protective than $47 \mathrm{II}(P=0.04)$.

The protective effect of KIR $3 D L 1$ I $47 V$ is apparent when taking into consideration alternative outcome measures. We next used a mixed linear-effects model to estimate the effect of copy numbers of KIR3DL1 47V on longitudinally measured HIV VL and CD4 counts, adjusting for allelic effects of $H L A-A, H L A-B$, and $H L A-C$ and the post-enrollment timing of VL measurements. The data were analyzed for all subjects combined, for those who were $H L A-B$ $B w 4^{+} / B^{\star} 57$, and for $B^{\star} 57$-only subjects, for whom KIR3DS1 and $K I R 3 D L 1^{*} 004$ were taken into account by being coded as random variables (Supplemental Table 1). Consistent with the above results, the strongest effect on longitudinal VLs was seen in $B^{*} 57^{+}$individuals, for whom increasing copy numbers of KIR3DL1 $47 \mathrm{~V}$ were associated with a significantly lower VL $\left(-0.14 \log _{10}\right.$ copies/ml per KIR3DL1 $47 \mathrm{~V}$ allele, $P=4.9 \times 10^{-18}$; Table 2$)$. The protective effect of $47 \mathrm{~V}$ was also observed when considering the $\mathrm{mVL}\left(-0.18 \log _{10}\right.$ copies $/ \mathrm{ml}$ per KIR3DL1 $47 \mathrm{~V}$ allele, $\left.P=6.3 \times 10^{-4}\right)$ and $C D 4^{+} \mathrm{T}$ cell counts, a marker of immunopathology due to HIV infection (increase of 24.88 cells/ $\mu$ l per KIR3DL1 $47 \mathrm{~V}$ allele, $P=1.5 \times 10^{-6}$; Table 2). In stark contrast, there was a significant detrimental effect of increasing copy numbers of KIR3DL1 $47 \mathrm{~V}$ among $B w 4^{+} / B^{\star} 57^{-}$subjects, suggesting overt differential consequences attributable to the fine specificity of KIRHLA allotypic interactions on NK cell function.

\section{Table 2. Effect of KIR3DL1 I47V on various outcomes ${ }^{\mathrm{A}}$}

\begin{tabular}{|c|c|c|c|c|c|}
\hline & Outcome measure & $n(I I / I V / V V)$ & Effect $^{\mathrm{B}}$ (linear estimate) & SE & $P$ value \\
\hline All & Longitudinal $\log _{10} \mathrm{VL}$ & $3,865(1,309 / 1,800 / 756)$ & 0.003 & 0.008 & 0.69 \\
\hline$H L A-B B w 4^{+} / B^{*} 57^{-}$ & Longitudinal $\log _{10} \mathrm{VL}$ & 2,022 (659/958/405) & 0.09 & 0.012 & $2.2 \times 10^{-13}$ \\
\hline$B^{*} 57^{+}$ & Longitudinal $\log _{10} \mathrm{VL}$ & $674(238 / 306 / 130)$ & -0.14 & 0.016 & $4.9 \times 10^{-18}$ \\
\hline$B^{*} 57^{+}$ & Longitudinal CD4+ $T$ cell count & $588(208 / 272 / 108)$ & 24.88 & 4.13 & $1.5 \times 10^{-6}$ \\
\hline
\end{tabular}

${ }^{A} H L A-A,-B$, and $-C$ alleles, timing of VLs measurements, as well as the presence of KIR3DL1*004 and KIR3DS1 were taken into account by being coded as random effects for all analyses using a mixed linear-effects model. ${ }^{B}$ Effect estimates denote the effect of each additional $3 D L 147 V$ as an additive effect. 
Table 3. Effect of KIR3DL1 I47V on log VL and CD4+ $T$ cell counts in individuals with $B * 57: 01$ and $B * 57: 03^{A}$

$\begin{array}{lccccc}\text { Genotype } & \boldsymbol{n} & \text { Outcome } & \text { Effect }^{\mathrm{B}} \text { (linear estimate) } & \text { SE } & \boldsymbol{P} \text { value } \\ B^{*} 57: 01^{+} & 398 & \text { Longitudinal } \log _{10} \mathrm{VL} & -0.36 & 0.02 & 1.7 \times 10^{-67} \\ B^{*} 57: 03^{+} & 245 & & 0.01 & 0.03 & 0.16 \\ B^{*} 57: 01^{+} & 383 & \text { Longitudinal } \mathrm{CD}^{+}{ }^{+} \text {T cell count } & 90.9 & 6.53 & 4.8 \times 10^{-43} \\ B^{*} 57: 03^{+} & 241 & & 20 & 9.4 & 0.06\end{array}$

${ }^{A} H L A-A,-B$, and $-C$ alleles, timing of VL measurements, as well as the presence of $K I R 3 D L 1^{*} 004$ and $K I R 3 D S 1$ were taken into account by being coded as random effects for all analyses using a mixed linear-effects model. BEffect estimates denote the effect of each additional 3DL147V as an additive effect.

interface (Supplemental Figure 3A), we hypothesized that these positions would not directly affect the binding affinity of the KIR3DL1 receptor on NK cells. Nevertheless, to better understand how polymorphisms at positions 2, 47, and 54 impact recognition of the peptideHLA-B ${ }^{\star}$ 57:01 complex, we initially used surface plasmon resonance (SPR) to compare direct $1: 1$ binding of a number of $\mathrm{B}^{\star} 57$ :01-peptide complexes with KIR3DL1*015 $(2 \mathrm{~V} / 47 \mathrm{~V} / 54 \mathrm{~L})$ or KIR3DL1 ${ }^{*} 001$ and

The effect of KIR3DL1 I47V variation on HIV control is specific to $B^{\star} 57: 01$ and requires surface-expressed KIR3DL1 alleles. In an attempt to further explore the specificity of the KIR3DL1 $I 47 V-B^{\star} 57$ interaction, we next analyzed individual $B^{\star} 57$ subtypes. This analysis was restricted to $B^{\star} 57: 01$ and $B^{\star} 57: 03$, because we had insufficient power to determine a reliable effect estimate for $B^{\star} 57: 02$ and $B^{\star} 57: 04$ ( $n=28$ and 3, respectively). Reminiscent of abacavir specificity for $B^{\star} 57: 01$ (29-32), the protective effect of KIR3DL1 I $47 \mathrm{~V}$ on the longitudinal VL was only observed among individuals with $B^{\star} 57: 01\left(-0.36 \log _{10}\right.$ copies $/ \mathrm{ml}, P=1.7 \times 10^{-67}$; Table 3), with no effect seen for $B^{\star} 57: 03\left(0.01 \log _{10}\right.$ copies $/ \mathrm{ml}$, $P=0.16)$. We observed similar results for longitudinal $\mathrm{CD}^{+} \mathrm{T}$ cell counts $\left(90.9\right.$ cells $/ \mu \mathrm{l}, P=1.7 \times 10^{-43}$ for $B^{\star} 57: 01$ vs. 20 cells $/ \mu 1, P=$ 0.06 for $B^{\star} 57: 03$; Table 3 ), as well as for the comparison of controllers versus noncontrollers $\left(B^{\star} 57: 01 \mathrm{VV}\right.$ vs. II: $\mathrm{OR}=0.2, P=0.001$; $B * 57: 03$ VV vs. II: $\mathrm{OR}=0.4, P=0.25$; data not shown).

We estimated the effect of each common KIR3DL1 allele (see Supplemental Table 3) on longitudinal viremia among $B^{\star} 57^{+}$individuals in order to compare association patterns between $B^{\star} 57: 01$ and $B^{*} 57: 03$ as a function of KIR3DL1 subtype. The $47 \mathrm{~V}$ alleles KIR3DL $1^{*} 015$ and $K I R 3 D L 1^{*} 002$ were associated with significantly lower VLs, whereas the $47 I$ allele $K I R 3 D L 1^{\star} 001$ was associated with a higher VL among $B^{*} 57$ :01 subjects (Figure $2 A$ ). KIR3DL1 ${ }^{\star} 015$ $(2 \mathrm{~V} / 47 \mathrm{~V} / 54 \mathrm{~L})$ and KIR3DL1 ${ }^{*} 001(2 \mathrm{M} / 47 \mathrm{I} / 54 \mathrm{I})$ have identical aa sequences, except for positions 2, 47, and 54 (Figure 2C), and it is therefore notable that these 2 alleles modify $B^{*} 57: 01$ effects on HIV in opposite directions. In $B^{\star} 57: 03^{+}$subjects, a distinct pattern of association was observed, in which KIR3DL1*002 (47V) was associated with a high VL and $K I R 3 D L 1^{\star} 001$ (47I) with protection (Figure 2B). KIR3DL1*004 (47I allele), which is not expressed at the cell surface (25), showed no effect, which is consistent with the protective effect of $47 \mathrm{~V}$ being specific to KIR3DL1 (as opposed to it tagging variants in neighboring genes) and requiring a surfaceexpressed KIR3DL1 in conjunction with $\mathrm{B}^{\star} 57: 01$. $\mathrm{B}^{\star} 57: 01$ and $\mathrm{B}^{\star} 57: 03$ differ by only 2 aa: 114 (D vs. N, respectively) and 116 (S vs. Y, respectively), located in the $\mathrm{E}$ and $\mathrm{F}$ pockets, respectively, of the peptide-binding groove, which are key pockets for determining which peptides will bind (30). These data raise the possibility that the presentation of peptide bound to $\mathrm{B}^{\star} 57$ molecules may determine, at least in part, whether or not $47 \mathrm{~V}$ modulates protection conferred by the specific $\mathrm{B}^{\star} 57$ subtype.

The effect of KIR3DL1 I47V on ligand binding. Given the distal location of positions 2,47 , and 54 to the peptide-HLA-binding
KIR3DL1 ${ }^{*} 005(2 \mathrm{M} / 47 \mathrm{I} / 54 \mathrm{I})$. In spite of the clearly opposing effects of KIR3DL1 ${ }^{*} 015$ and KIR3DL1 ${ }^{*} 001$ on modification of $\mathrm{B}^{\star} 57: 01$ protection (Figure $3 \mathrm{~A}$ ), these $2 \mathrm{KIR}$ allotypes bound to $\mathrm{B}^{\star} 57$ :01 with similar affinity, but with slightly weaker affinity than binding to KIR3DL1*005. This confirmed that there was no strict correlation between $47 \mathrm{~V}$-associated protection and a higher affinity of $47 \mathrm{~V}$ KIR3DL1 allotypes for $B^{\star} 57: 01$, at least with the peptides tested via SPR (Supplemental Figure 3, B-E, and Supplemental Table 5).

Since we did not observe differences in affinity with SPR, positions 2, 47, and 54 might alter the avidity of KIR3DL1 through receptor clustering. On the basis of the crystal packing of the KIR3DL1-B57:01 complex (33), we constructed a structural model of KIR3DL1 (Supplemental Figure 3A), in which positions 47 and 54 are part of an extensive interface incorporating the $\alpha 2$ and $\alpha 3$ domains of neighboring HLA. This region is strictly conserved in $B^{*} 57$ allotypes and highly conserved across HLA alleles (IMGT/ HLA database: https://www.ebi.ac.uk/ipd/imgt/hla/). In this model, each KIR3DL1 molecule thus makes contact with 2 HLA molecules to form higher-order oligomers (i.e., clustering).

To further probe multimeric interactions between KIR3DL1HLA, we assessed the binding of HLA- $^{\star}{ }^{\star 08: 01 ~(n e g a t i v e ~ c o n t r o l) ~}$ and $B^{\star} 57: 01$ tetramers to $293 \mathrm{~T}$ cells expressing FLAG-tagged KIR3DL1 ${ }^{*} 001$ or KIR3DL1 ${ }^{\star} 015$ (which differ only at positions 2, 47 , and 54) or to mutant KIR3DL1 molecules containing reciprocal mutations at each position. Analyses of $\mathrm{B}^{*} 57$ tetramer binding to cells expressing matched levels of KIR3DL1, as defined by equivalent staining with an anti-FLAG $\mathrm{mAb}$, showed increased binding to KIR3DL1 ${ }^{\star} 001$ compared with binding to KIR3DL1 ${ }^{\star} 015$ (Figure 3). Mutation of position 47 in the context of either KIR3DL1 ${ }^{*} 001$ or KIR3DL1 ${ }^{*} 015$ had little impact on tetramer binding, whereas mutation of residue 54 resulted in an intermediate phenotype, subtly changing binding of the $\mathrm{B}^{\star} 57$ tetramer to both KIR3DL1 ${ }^{\star} 001$ and KIR3DL1 ${ }^{*} 015$. In contrast, substitution of $\mathrm{M}$ with $\mathrm{V}$ at position 2 of KIR3DL $1^{*} 001$ resulted in impaired binding, similar to that observed for $\mathrm{KIR} 3 \mathrm{DL} 1^{\star} 015$, but the reciprocal mutation in KIR3DL1 ${ }^{*} 015$ had no effect, suggesting that its effect was dependent on the presence of 47I and/or 54I. Taken together, these data suggest that the dimorphisms at these 3 positions have little direct effect on affinity, consistent with their location distant from the ligand-binding site of KIR3DL1 (Supplemental Figure 3A); yet in the context of multivalent binding, these residues may act coordinately in a manner that impacts the recognition of $\mathrm{B}^{*} 57$, possibly affecting receptor clustering and thereby affecting NK cell function. 


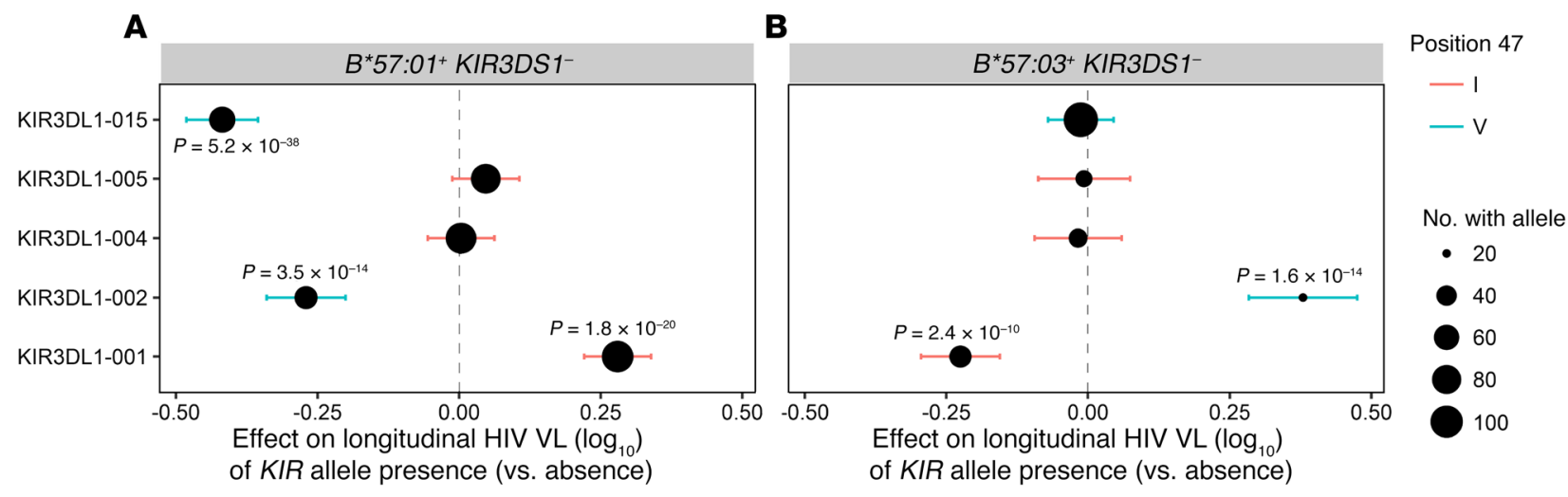

C

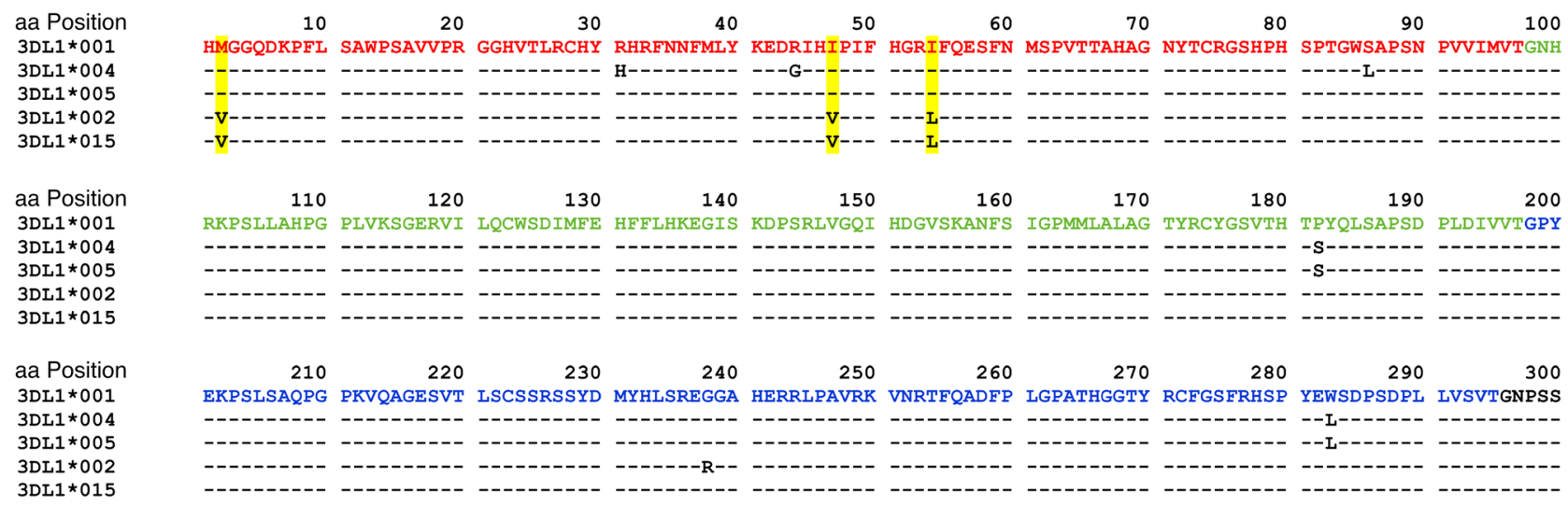

Figure 2. Effect of individual KIR3DL1 alleles on longitudinal HIV viremia in subjects with $B * \mathbf{5 7}$ subtypes. A mixed linear-effects model adjusting for all $H L A-A,-B$, and $-C$ alleles and post-enrollment timing of measurements was used to estimate the effect of each KIR3DL 1 allele for groups including individuals with (A) $B^{*} 57: 01$ only and (B) $B^{*} 57: 03$ only. $47 \mathrm{I}$ alleles are shown in red, and 47V alleles are shown in blue. The horizontal bars represent a $95 \% \mathrm{Cl}$. The size of the black dots was scaled according to the frequency of each allele. Alleles with estimates that did not cross the line, which represents no change in VL (0), are significant. (C) Extracellular domain aa alignment of the $K I R 3 D L 1$ alleles tested in $\mathbf{A}$ and $\mathbf{B}$ (http://www.ebi.ac.uk/ipd/kir/). Red = DO, green = D1, blue = D2. Positions 2, 47, and 54 are highlighted in yellow.

\section{Discussion}

Superior HIV control among the $B^{\star} 57^{+}$subjects as a function of enhanced CTL responses against $B^{\star} 57$-restricted epitopes and the $\mathrm{T}$ cell receptors that recognize the complex $(12-14,21,34)$ cannot explain the sizeable heterogeneity in HIV disease progression observed in the $B^{*} 57^{+}$subjects. Analyses of our European American seroconverter cohorts showed that $50 \%$ of $B^{\star} 57^{+}$subjects had progressed to AIDS 1993 (1993 definition of the US Centers for Disease Control and Prevention) (35) by 11.2 years after infection compared with $71 \%$ of $B^{\star} 57^{-}$subjects (data not shown). $B^{\star} 57^{+}$controllers and noncontrollers present the same immunodominant $B^{\star} 57$-restricted HIV gag epitopes, and escape mutations can occur in both groups (36). Thus, modifiers of $\mathrm{B}^{\star} 57$ responses against HIV probably affect its efficacy.

We used a genome-wide approach to examine genetic variants that may enhance or diminish $B^{\star} 57$ protection among controllers versus noncontrollers and identified only a single variant encoding an isoleucine-to-valine change at position 47 in the KIR3DL1 molecule. The association was conclusively verified in a second cohort of viremic controllers compared with noncontrollers $(P$ $=0.004)$ and in an analysis of longitudinal VLs and CD $4^{+} \mathrm{T}$ cell counts $\left(P=4.9 \times 10^{-18}\right.$ and $1.5 \times 10^{-6}$, respectively), with each additional $3 D L 147 \mathrm{~V}$ as an additive effect.
Stronger KIR3DL1-mediated inhibition of NK cells in the presence of HLA-B Bw4 subtypes that have isoleucine at position 80 (Bw4-80I) relative to those with threonine (Bw4-80T) (37) was the first indication of differential binding of KIR3DL1 to its various HLA-Bw4 ligands. Additional nuances in differential KIR3DL1HLA-B interactions have since been reported, including recent studies suggesting a variation in surface expression of both receptor and ligand (38) as well as a differential binding capacity of specific KIR3DL1 allotypes to certain HLA-Bw4 ligands (24, 25, 38-40). High cell-surface-expression alleles of KIR3DL1 in combination with $H L A-B B w 4-80 I$ alleles, which include $B{ }^{\star} 57$, were previously shown to be protective against HIV-1 (27). While there is overlap between $47 \mathrm{~V}$ and high-expression alleles (and between lowexpression alleles and 47I) (Supplemental Figure 2), the groupings are not identical. Most notably, KIR3DL1 ${ }^{\star} 001$ is a high-expression 47I allele, and KIR3DL1 ${ }^{*} 007$ is a low-expression 47V allele. The modifying effect of $47 \mathrm{~V}$ appears to be $\mathrm{B}^{\star} 57: 01$ specific, whereas the effect of high/low KIR3DL1 expression was observed for $H L A-B$ $B w 4-80 I$ alleles overall. In light of our previous findings regarding KIR3DL1 expression levels (27), as well as the protective effect of KIR3DS1 in combination with HLA-B Bw4-8OI (which includes $B^{\star} 57$ ) (26), we performed a multivariate mixed linear-effects analysis to determine the relative effects of KIR3DL1 aa47, KIR3DL1 

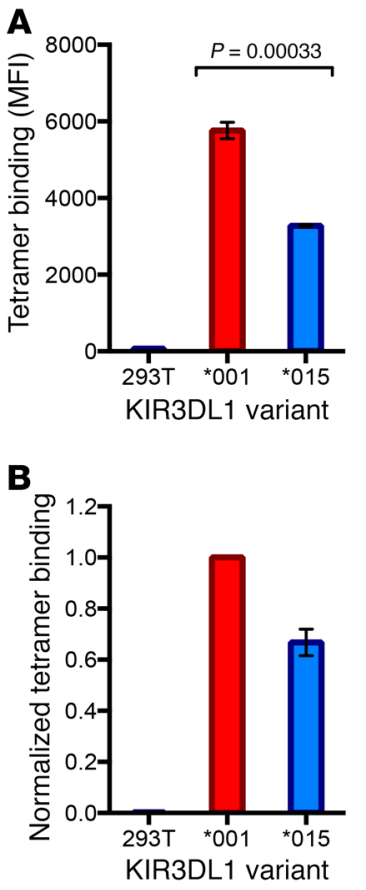
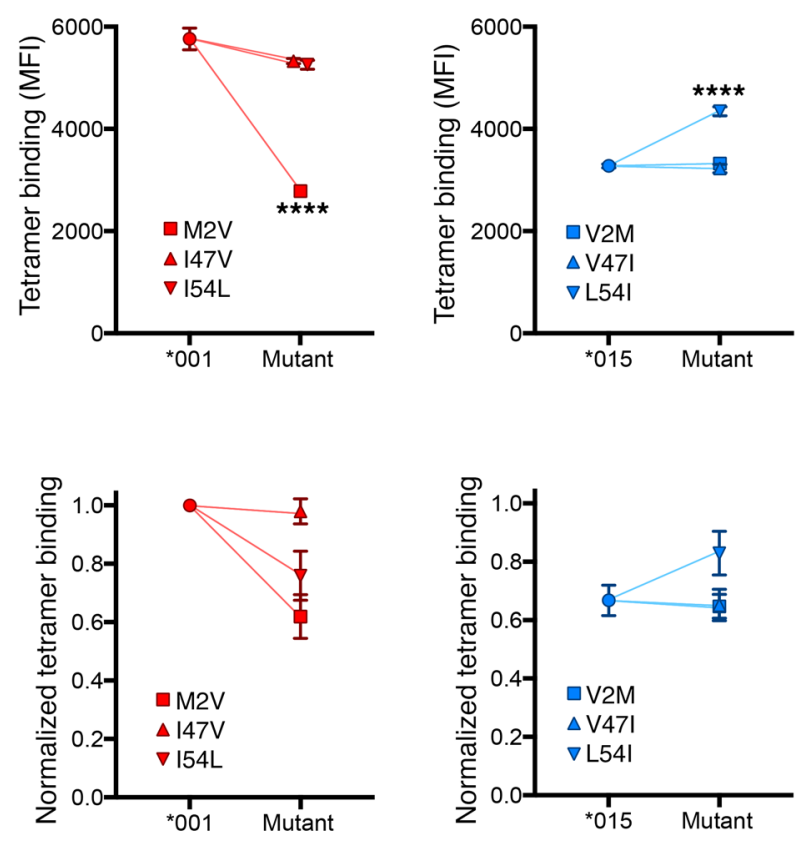

Figure 3. HLA-B*57:01/LF9 tetramer binding to KIR3DL1 variants. 293T cells were transfected with FLAG-tagged KIR3DL1*001, KIR3DL1*015, or mutants and stained with HLA-B* $57: 01 /$ LF9 or HLA-B*08:01/FLR tetramers (350 ng each). (A) MFI of HLA-B* $57: 01 /$ LF9 binding to FLAG-positive sections from a representative experiment (performed in triplicate). ${ }^{* * *} P<0.0001$, by ANOVA with Tukey's multiple comparisons test. Data represent the mean \pm SEM. (B) The MFI of tetramer staining on FLAG-positive sections was normalized to HLA-B*57:01/LF9 binding to KIR3DL1*001 across 4 independent transfection experiments. Data represent the mean $\pm \mathrm{SEM}$. expression levels, and KIR3DS1 on HIV viremia in $B^{\star} 57: 01^{+}$and $B^{\star} 57: 03^{+}$subjects (see Supplemental Table 6). For $B^{\star} 57: 01$, we found that the strongest and most significant KIR3D effect involved KIR3DL1 47V (effect estimate $=-0.53$ for each additional $47 \mathrm{~V}$ copy; $\left.P=9 \times 10^{-86}\right)$. Nevertheless, some $B^{\star} 57: 01^{+}$individuals with the protective KIR3DL1 $47 \mathrm{~V}$ allele were noncontrollers, supporting a model in which a complex set of host and viral factors determine disease outcome among $B^{\star} 57: 01^{+}$subjects, with KIR3DL1 $47 \mathrm{~V}$ being one of these factors. Variation in other regions of the genome that are difficult to decipher by GWAS, such as genes encoding T cell receptors, immunoglobulins, and the leukocyte receptor complex (which includes the KIR genes), along with viral, environmental, and behavioral factors, are likely to affect the ability of $B^{\star} 57^{+}$subjects to control HIV. Overall, the impact of KIR3DL1 variation on HIV outcome is multifactorial and characterized by fine specificity between KIR3DL1 allotypes and their HLA-B + peptide ligands, which likely affects the NK cell response to HIV-infected cells.

Two common alleles containing 47V, KIR3DL1 ${ }^{*} 015$ and KIR $3 D L 1^{*} 002$ confer strong protection in combination with $B^{\star} 57: 01$, and these $K I R$ alleles probably account, in large part, for the observed effect of $47 \mathrm{~V}$ among $B^{\star} 57: 01^{+}$subjects (Figure 2). KIR3DL1 ${ }^{*} 015$ and KIR3DL1 ${ }^{*} 002$ differ only at a single aa in the D2 domain, which is unlikely to alter the interaction with $\mathrm{B}^{\star} 57: 01$. However, KIR3DL1 ${ }^{*} 015$ and KIR3DL1 ${ }^{*} 001$, which differ only at aa 2, 47, and 54 (Figure 2C), convincingly showed opposite modifying effects on $B^{\star} 57: 01$. This genetic epidemiological observation may be related to the reduction in binding of $B^{\star} 57: 01$ tetramers to KIR3DL1 ${ }^{*} 015$ relative to KIR3DL1 ${ }^{*} 001$ and KIR3DL1 ${ }^{*} 005$ (ref. 41 and Figure 3). Perhaps HIV Nef downregulation of HLA-B, in

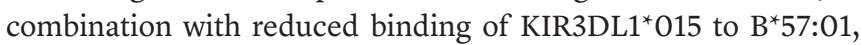
results in attenuated inhibitory signaling through this KIR, resulting in greater $\mathrm{NK}$ cell activation against HIV-infected targets. Both HLA-B ${ }^{\star} 57: 01$ and $B^{\star} 57: 03$ bind KIR3DL1 with high affinity as assessed by their binding to bead-immobilized HLA class I allotypes. Indeed, HLA-B ${ }^{\star} 57: 01$ has been the strongest KIR3DL1-binding HLA allotype, irrespective of the KIR3DL1 allele tested, and $B^{*} 57: 03$ has typically been in the top $10 \%$ of allotypes (40). As such, they would both be expected to license KIR3DL1 ${ }^{+}$ NK cells effectively, but there are no data to confirm whether the small incremental increase in KIR3DL1 binding shown in our study results in a difference in the number of licensed cells between $\mathrm{B}^{\star} 57: 01^{+}$and $\mathrm{B}^{*} 57: 03^{+}$individuals.

In spite of differential tetramer binding to KIR3DL1 ${ }^{\star} 001$ (47I) versus KIR3DL1 ${ }^{*} 015(47 \mathrm{~V})$, we did not detect clear differences in binding affinities of KIR3DL1 ${ }^{*} 001$ or KIR3DL $1 * 015$ for $\mathrm{B}^{\star} 57$ :01 by SPR (Supplemental Figure 3, B-E, and Supplemental Table 5). As these experiments were conducted with a limited number of peptides, any differences in affinity for $\mathrm{B}^{\star} 57$ :01 conferred by positions 2,47 , and 54 may be apparent only across a diverse repertoire of peptides. It is also possible that positions 2,47 , and 54 may impact receptor clustering on NK cells and thus the avidity of the interaction. Differences in receptor clustering on the cell surface, a surrogate for signaling potential, may thereby alter the NK cell response to HIV-infected targets. Positions 2, 47, and 54 also flank sites in D0 (positions 5, 31, and 32) that have been shown to be under positive natural selection (42), and there are data that further support the importance of these 3 positions in ligand binding (23).

Individually, the impact of mutations at positions 2,47 , or 54 of KIR3DL1 differed markedly, but each was dependent on the variants present at the other 2 positions (Figure 3), indicating that any potential effect of a given polymorphism is dependent on the structural framework of the DO domain. Furthermore, mutagenesis experiments indicate that DO is important for folding and cell-surface expression of KIR3DL1 (43) and contributes to the binding avidity of HLA ligands (44). Limited aa differences across KIR ligands can also impact the consequences of their interactions. $\mathrm{B}^{\star} 57: 01$ and $\mathrm{B}^{\star}$ 57:03 differ by 2 aa (positions 114 and 116) in the peptide-binding groove, and although their peptide-binding 
motifs are nearly indistinguishable, they do show some differences in peptide preference (HIV Molecular Immunology Database: hiv. lanl.gov), peptide positioning and presentation, and immune pressure on the virus. Crystal structures of HLA-B ${ }^{*} 57: 01$ and $B^{*} 57: 03$ have been previously determined by us and other groups $(29,30$, 33, 45). Positions 114 (aspartate to asparagine) and 116 (serine to tyrosine) lie in the floor of the peptide-binding groove and hence cannot directly contact KIR3DL1. Rather, they alter the architecture and charge of the $\mathrm{E}$ and F pockets. Thus, they probably affect the repertoire of peptides that can bind each allotype and the way in which peptides are positioned in the binding groove, thereby differentially altering the recognition of these peptide-MHC complexes by any given KIR3DL1 subtype. Recent data also indicate that KIR3DL1 polymorphisms contribute to differential binding preferences for HLA class I allotypes, with $B^{*} 57: 01$ showing better binding than $B^{*} 57: 03$ (40). These distinctions profoundly influence the modifying effects of specific KIR3DL1 alleles on the closely related $B^{*} 57: 01$ versus $B^{*} 57: 03$ alleles (Figure 2 ) and emphasize the granularity of KIR3DL1-HLA-B interactions.

\section{Methods}

Subjects. Data from a total of 3,878 HIV-1-infected individuals, including $818 B^{*} 57^{+}$individuals ( $n=482$ whites, $n=270$ blacks, $n=40$ other, $n=26$ unknown), were used in this study. The $B^{*} 57^{+}$individuals included 614 men (75.1\%), 150 women (18.3\%), 4 transgender individuals (0.5\%), and 50 individuals of unknown gender (6.1\%). In the discovery phase, WGS was performed on $100 B^{*} 57^{+} \mathrm{HIV}^{+}$controllers $(n=90$ whites, $n=10$ other/unknown) and $100 B^{*} 57^{+} \mathrm{HIV}^{+}$noncontrollers $(n=97$ whites, $n=3$ other/unknown). Controllers were antiretroviral therapynaive (ART-naive) individuals with at least $3 \mathrm{VL}$ measurements (most individuals had $>6$ ), the majority of which had fewer than 500 copies of HIV-1 RNA/ml of plasma (range $=<500-1,681 ; 89 \%$ had $<500) ; \mathrm{CD}^{+}$ T cell counts above $400 / \mathrm{mm}^{3}$; and a duration of infection of more than 4 years. Noncontrollers included individuals with at least $1 \mathrm{VL}$ above 10,000 copies/ml of plasma and at least $1 \mathrm{CD}^{+} \mathrm{T}$ cell count below $500 / \mathrm{mm}^{3}$ (most had multiple CD4 counts of $<500 / \mathrm{mm}^{3}$ ).

The numbers for $B^{*} 57^{+}$individuals used in each outcome analysis are shown in Supplemental Table 1. For the mixed linear-effects analyses, a total of 36,074 longitudinal VL measurements were available for 3,865 individuals of all $H L A-B$ genotypes (individuals with $\geq 1 \mathrm{VL}$ measurement), including 17,548 measurements for $674 B^{*} 57^{+}$individuals. A total of $16,050 \mathrm{CD} 4$ counts were available for $588 B^{*} 57^{+}$individuals. There was an overlap of $566 B^{*} 57^{+}$subjects between the categorical analyses $(n=710)$ and the mixed linear-effects analyses $(n=674)$.

The subjects in this study were enrolled into 1 of 8 study cohorts: the Multicenter AIDS Cohort Study (MACS) (46); the AIDS linked to the Intravenous Experience (ALIVE) cohort (47); the Massachusetts General Hospital (MGH) controller cohort (http://hivcontrollers.org/ hivcontrollers/); the Study on the Consequences of Protease Inhibitor Era (SCOPE) (48); the AIDS Clinical Trial Group (ACTG) cohort (https://actgnetwork.org); the Swiss HIV Cohort Study (SHCS) (http:// www.shcs.ch); the U.S. military HIV Natural History Study (USMNHS) (www.idcrp.org); and the NIAID long-term nonprogressor cohort (49).

WGS. WGS data were generated for 191 of $200 \mathrm{~B}^{*} 57^{+}$samples on the Illumina HiSeq 2000 platform using 100-bp paired-end reads. Samples were sequenced to an average depth of $36 \times$ coverage (range 29.9-53.4×). PCR duplicates were removed using Picard Tools, version
1.59 , and unique reads were aligned to build 37 of the human genome reference sequencing using BWA, version 0.5.10. Sequence variants were identified using GATK-1.6-11 and annotated using snpEff-3.3.

Two samples were found to have discordances between their self-declared gender and that inferred from the sequencing data using the estimated $\mathrm{X} / \mathrm{Y}$ chromosome ratios and were excluded from further analysis. Two additional samples were found to be duplicates based on $100 \%$ identity using a panel of 4,000 common variants to test for cryptic relatedness between samples and were also excluded. Thus, 187 samples were included in the genomewide analysis, of which 97 were from controllers and 90 were from noncontrollers.

Associations between genetic variants and controller or noncontroller status were examined using both single-variant tests and genebased collapsing analyses of rare functional variants (minor allele frequency $<5 \%$ ) using the Analysis Tool for Annotated Variants (ATAV) software package (https://github.com/igm-team/atav). Significance scores were determined using Fisher's exact test adjusted for multiple testing using a Bonferroni correction for the number of variants or genes included in each analysis.

HLA and KIR genotyping. HLA genotyping was performed either by PCR-sequence-specific oligonucleotide probing (PCR-SSOP), PCR-sequence-based typing (PCR-SBT) using the Sanger sequencing technology recommended by the $13^{\text {th }}$ International Histocompatibility Workshop (http://www.ihwg.org), or next-generation sequencing using the Roche 454 platform (50). KIR genotyping for the presence or absence of KIR genes was performed by PCR-sequence-specific priming (PCR-SSP) (51), followed by KIR3DL1 sequencing using genespecific primers (52). Allele frequencies are shown in Supplemental Figure 2 and Supplemental Table 3. These frequencies are comparable with those seen in the general population (http://www.allelefrequencies. net/default.asp). The KIR3DL1 copy number was determined using a quantitative real-time PCR assay as previously described (28). In cases in which DNA was not available, the copy number was imputed on the basis of the overall KIR profile and the results of KIR3DL1 subtyping.

Protein expression and purification. The HLA-B ${ }^{\star} 57: 01$ and $\beta 2$ microglobulin genes were subcloned into the pET-30 expression vector and expressed separately into inclusion bodies in E. coli. The HLA complex was refolded in the presence of the ISPRTLNAW (IW9, HIV-1 gag), KAFSPEVIPMF (KF11, HIV-1 gag), QASQEVKNW (QW9, HIV-1 gag), and LSSPVTKSF (LF9, self) peptides and purified as described previously (53). KIR3DL1 ${ }^{*} 001$, KIR3DL1 ${ }^{*} 005$, and KIR3DL1*015 (residues 1-299) were subcloned into the pFastBac insect expression vector with $\mathrm{N}$-terminal $6 \mathrm{xHis}$ and secretion tags. KIR3DL1 was expressed from Hi-5 insect cells (Invitrogen, Thermo Fisher Scientific) and secreted into the culture media. The protein was purified as described previously (33) and then concentrated in a buffer composed of $10 \mathrm{mM}$ Tris (pH 8.0) and $300 \mathrm{mM} \mathrm{NaCl}$, prior to use in SPR experiments.

$S P R$. SPR experiments were used to measure the affinities of KIR3DL1 ${ }^{*} 001$, KIR3DL1 ${ }^{*} 005$, and KIR3DL1 ${ }^{*} 015$ for HLA-B ${ }^{*} 57: 01$ presenting the HIV-1 gag epitopes IW9, KF11, and QW9 and the self-epitope LF9. SPR experiments were conducted on a Biacore 3000 instrument at $298 \mathrm{~K}$. A CM5 chip was used to immobilize the antiHLA mAb W6/32 (generated in-house, ref. 54) via amine coupling. HLA-B 57 :01-peptide complexes were then captured on the chip by binding to W6/32 to a surface density of approximately 700 response units. KIR3DL1*001 (2.25-350 $\mu \mathrm{M})$, KIR3DL1*005 $(2.25-350 \mu \mathrm{M})$, and KIR3DL1*015 $(1.56-200 \mu \mathrm{M})$ were injected over the chip at a 
flow rate of $5 \mu \mathrm{l} / \mathrm{min}$ in a buffer composed of $10 \mathrm{mM}$ HEPES-HCl $\mathrm{pH}$ 7.4), $150 \mathrm{mM} \mathrm{NaCl}$, and $0.005 \%$ surfactant P20. The response to the W6/32-only control flow cell was subtracted from the responses to HLA-B ${ }^{\star}$ 77:01-peptides. Equilibrium data were analyzed using GraphPad Prism Version 7. All data are representative of 2 independent experiments, with each experiment conducted using duplicate samples. Data represent the mean \pm SEM.

Tetramer-binding assay. pEF6.FLAG-tagged KIR3DL1*001 and KIR3DL1 ${ }^{*} 015$ constructs (41) were mutated at residues 2,47 , and 54 via site-directed mutagenesis. pEF6.KIR3DL1 ${ }^{*} 001$ and KIR3DL1 ${ }^{*} 015$ were transfected into 293T cells using FuGENE 6 Transfection Reagent (Promega) according to the manufacturer's instructions, and KIR3DL1 surface expression was confirmed via staining with NKB1FITC (clone DX9; BD Pharmingen) after 48 hours. Transfected 293T cells (originally purchased from ATCC) were stained with $350 \mathrm{ng} / \mathrm{test}$ tetrameric HLA-B*57:01/LF9 or HLA-B*08:01/FLR conjugated with phycoerythrin-streptavidin (PE-SA) and incubated at room temperature for 30 minutes. Cells were washed and stained with anti-FLAGAPC (clone L5; BioLegend) before flow cytometric analysis.

Statistics. SAS 9.2 (SAS Institute) or R 3.3.1 (The R Foundation) was used for data management and statistical analyses. PROC FREQ was used to compute frequencies on categorical variables. PROC LOGISTIC was used to calculate odds ratios and 95\% CIs. These analyses were performed with combined white, black, and other races/ethnicities (Hispanic/Latino, Asian), adjusting for race. Statistical significance refers to 2-sided $P$ values of less than 0.05 .

Analyses of the longitudinal VLs and $\mathrm{CD} 4^{+} \mathrm{T}$ cell counts were performed using the lmer function in $\mathrm{R}$. We allowed for random effects due to each $H L A-A, H L A-B$, and $H L A-C$ allele (or where modified, as specified in Results), the post-enrollment timing of measurements, and a correction for diploid $H L A$ allele coding. Several alternative outcomes were tested, including the $\log _{10}$-transformed HIV VL at each time point, a geometric mean HIV VL (mVL) of all time points, and the $\mathrm{CD} 4^{+} \mathrm{T}$ cell count at each time point. Likelihood ratio $P$ values were calculated using the ANOVA function in $\mathrm{R}$ in order to compare nested models fit under a maximum likelihood scenario. The $P$ values reported were not adjusted for multiple comparisons.

Study approval. This study was reviewed and approved by the protocol review office of the NCI Office of Human Subjects Research Protections (OHSRP) in Bethesda, Maryland. Informed consent was obtained at the study sites from all individuals prior to their participation in the study.

\section{Author contributions}

MC designed the study. MC, MPM, and VN wrote the manuscript. MPM, PRS, VR, XG, PP, PMS, JW, SCW, DAP, BAL, EG and SLL contributed to experiments. VN, YQ, NV, JPV, JR, AGB, ZLB, and JMC contributed to data analyses. RT coordinated sample selection for WGS. SMW, JJG, BDW, FPS, SGD, DWH, SAM, MC, NM, and JF contributed study cohort samples. MC, VN, MPM, JR, JPV, and AGB contributed to data interpretation. All authors contributed to the revision of the manuscript.

\section{Acknowledgments}

This article is dedicated to the memory of Dr. Bonnie Mathieson, a longtime friend and colleague who dedicated her career to the advancement of HIV research. We would like to thank Victoria
Walker-Sperling for her assistance with the figures. This work was supported by federal funds from the NCI, NIH, under contract HHSN261200800001E. The content of this publication does not necessarily reflect the views or policies of the Departments of the Army and Defense or Health and Human Services, nor does mention of trade names, commercial products, or organizations imply endorsement by the US Government. This Research was supported in part by the Intramural Research Program of the NIH, Frederick National Laboratory, Center for Cancer Research, and the National Health and Medical Research Council of Australia. Data in this manuscript were collected by the MACS and/or the Women's Interagency HIV Study (WIHS). The contents of this publication are solely the responsibility of the authors and do not represent the official views of the NIH. Data in this manuscript were collected by the Multicenter AIDS Cohort Study (MACS). MACS (Principal Investigators): Johns Hopkins University Bloomberg School of Public Health (Joseph Margolick, Todd Brown), U01-AI35042; Northwestern University (Steven Wolinsky), U01-AI35039; University of California, Los Angeles (Roger Detels, Otoniel Martinez-Maza, Otto Yang), U01-AI35040; University of Pittsburgh (Charles Rinaldo, Lawrence Kingsley, Jeremy Martinson), U01-AI35041; the Center for Analysis and Management of MACS, Johns Hopkins University Bloomberg School of Public Health (Lisa Jacobson, Gypsyamber D'Souza), UM1-AI35043. The MACS is funded primarily by the National Institute of Allergy and Infectious Diseases (NIAID), with additional co-funding from the National Cancer Institute (NCI), the National Institute on Drug Abuse (NIDA), and the National Institute of Mental Health (NIMH). Targeted supplemental funding for specific projects was also provided by the National Heart, Lung, and Blood Institute (NHLBI), and the National Institute on Deafness and Communication Disorders (NIDCD). MACS data collection is also supported by UL1-TR001079 (Johns Hopkins University Institute for Clinical and Translational Research [ICTR]) from the National Center for Advancing Translational Sciences (NCATS) a component of the National Institutes of Health (NIH), and NIH Roadmap for Medical Research. The contents of this publication are solely the responsibility of the authors and do not represent the official views of the National Institutes of Health (NIH), Johns Hopkins ICTR, or NCATS. The MACS website is located at http:// aidscohortstudy.org/. The ACTG was supported by the NIAID under NIH grants AI068636, AI038858, AI068634, AI106701 and AIO38855, and grants to Clinical Research Sites that participated in ACTG protocols and collected DNA under protocol A5128: AI069477, AIO27675, AI073961, AI069474, AI069432, AI069513, AI069423, AI050410, AI069452, AI69450, AI054907, AI069428, AI045008, AI069495, AI069415, AI069556, AI069484, AI069424, AIO69532, AI069419, AI069471, AIO25859, AI069418, AI050409, AI069501, AI069502, AI069511, AI069434, AI069465, AI069494, AI069472, AI069470, AIO46376, AI072626, AIO27661, AI034853, AI069447, AIO32782, AIO27658, AI27666, AI058740, AI046370, TR000445, RR00051, RR00046, RR025747, RR025777, RR024160, RR024996, and RR024156. Abbott Laboratories, Boehringer-Ingelheim, Bristol-Myers Squibb, Gilead Sciences, and GlaxoSmithKline provided study medications. DWH is also supported by NIH grants AIO77505, AI110527, and TR000445. JR is supported by the National Health and Medical Research Council of Australia, the Anti-cancer Council, the Austra- 
lian Research Council, and by an ARC Laureate Fellowship. ZLB is supported by a Scholar Award from the Michael Smith Foundation for Health Research. DAP is a Wellcome Trust Senior Investigator.
Address correspondence to: Mary Carrington, P.O. Box B, Bldg. 560, Rm 21-89, Frederick, Maryland 21702, USA. Phone: 301.846.1390; Email: carringm@mail.nih.gov.
1. Carrington M, O’Brien SJ. The influence of HLA genotype on AIDS. Annu Rev Med. 2003;54:535-551.

2. Fellay J, et al. A whole-genome association study of major determinants for host control of HIV-1. Science. 2007;317(5840):944-947.

3. International HIV Controllers Study, et al. The major genetic determinants of HIV-1 control affect HLA class I peptide presentation. Science. 2010;330(6010):1551-1557.

4. Gao X, et al. AIDS restriction HLA allotypes target distinct intervals of HIV-1 pathogenesis. Nat Med. 2005;11(12):1290-1292.

5. Kaslow RA, et al. Influence of combinations of human major histocompatibility complex genes on the course of HIV-1 infection. Nat Med. 1996;2(4):405-411.

6. Kiepiela P, et al. Dominant influence of HLA-B in mediating the potential co-evolution of HIV and HLA. Nature. 2004;432(7018):769-775.

7. Magierowska M, et al. Combined genotypes of CCR5, CCR2, SDF1, and HLA genes can predict the long-term nonprogressor status in human immunodeficiency virus-1-infected individuals. Blood. 1999;93(3):936-941.

8. Migueles SA, et al. HLA B ${ }^{*} 5701$ is highly associated with restriction of virus replication in a subgroup of HIV-infected long term nonprogressors. Proc Natl Acad Sci U S A. 2000;97(6):2709-2714.

9. Gao X, et al. Effect of a single amino acid change in MHC class I molecules on the rate of progression to AIDS. NEngl JMed. 2001;344(22):1668-1675.

10. Deeks SG, Walker BD. Human immunodeficiency virus controllers: mechanisms of durable virus control in the absence of antiretroviral therapy. Immunity. 2007;27(3):406-416.

11. Emu B, et al. HLA class I-restricted T-cell responses may contribute to the control of human immunodeficiency virus infection, but such responses are not always necessary for long-term virus control. J Virol. 2008;82(11):5398-5407.

12. Gillespie GM, et al. Cross-reactive cytotoxic T lymphocytes against a HIV-1 p24 epitope in slow progressors with $\mathrm{B}^{\star} 57$. AIDS. 2002;16(7):961-972.

13. Goulder PJ, et al. Novel, cross-restricted, conserved, and immunodominant cytotoxic $\mathrm{T}$ lymphocyte epitopes in slow progressors in HIV type 1 infection. AIDS Res Hum Retroviruses. 1996;12(18):1691-1698.

14. Kiepiela P, et al. CD8 ${ }^{+} \mathrm{T}$-cell responses to different HIV proteins have discordant associations with viral load. Nat Med. 2007;13(1):46-53.

15. Brockman MA, et al. Escape and compensation from early HLA-B57-mediated cytotoxic T-lymphocyte pressure on human immunodeficiency virus type $1 \mathrm{Gag}$ alter capsid interactions with cyclophilin A. J Virol. 2007;81(22):12608-12618.

16. Crawford H, et al. Evolution of HLA-B ${ }^{\star} 5703$ HIV-1 escape mutations in HLA-B ${ }^{*} 5703$-positive individuals and their transmission recipients. JExp Med. 2009;206(4):909-921.

17. Leslie AJ, et al. HIV evolution: CTL escape muta- tion and reversion after transmission. Nat Med. 2004;10(3):282-289.

18. Martinez-Picado J, et al. Fitness cost of escape mutations in p24 Gag in association with control of human immunodeficiency virus type 1. J Virol. 2006;80(7):3617-3623.

19. Miura T, et al. HLA-B57/B*5801 human immunodeficiency virus type 1 elite controllers select for rare gag variants associated with reduced viral replication capacity and strong cytotoxic T-lymphocyte [corrected] recognition. J Virol. 2009;83(6):2743-2755.

20. Carlson JM, et al. Impact of pre-adapted HIV transmission. Nat Med. 2016;22(6):606-613.

21. Turnbull EL, et al. HIV-1 epitope-specific $\mathrm{CD}^{+} \mathrm{T}$ cell responses strongly associated with delayed disease progression crossrecognize epitope variants efficiently. Jimmunol. 2006;176(10):6130-6146.

22. Kosmrlj A, et al. Effects of thymic selection of the T-cell repertoire on HLA class Iassociated control of HIV infection. Nature. 2010;465(7296):350-354.

23. Sharma D, et al. Dimorphic motifs in DO and D1+D2 domains of killer cell Ig-like receptor 3DL1 combine to form receptors with high, moderate, and no avidity for the complex of a peptide derived from HIV and HLA-A²402.JImmunol. 2009;183(7):4569-4582.

24. Gardiner CM, et al. Different NK cell surface phenotypes defined by the DX9 antibody are due to KIR3DL1 gene polymorphism. JImmunol. 2001;166(5):2992-3001.

25. Pando MJ, Gardiner CM, Gleimer M, McQueen KL, Parham P. The protein made from a common allele of KIR3DL1 (3DL1*004) is poorly expressed at cell surfaces due to substitution at positions 86 in Ig domain 0 and 182 in Ig domain 1. J Immunol. 2003;171(12):6640-6649.

26. Martin MP, et al. Epistatic interaction between KIR3DS1 and HLA-B delays the progression to AIDS. Nat Genet. 2002;31(4):429-434.

27. Martin MP, et al. Innate partnership of HLA-B and KIR3DL1 subtypes against HIV-1. Nat Genet. 2007;39(6):733-740.

28. Pelak K, et al. Copy number variation of KIR genes influences HIV-1 control. PLoS Biol. 2011;9(11):e1001208.

29. Chessman D, et al. Human leukocyte antigen class I-restricted activation of $\mathrm{CD} 8^{+} \mathrm{T}$ cells provides the immunogenetic basis of a systemic drug hypersensitivity. Immunity. 2008;28(6):822-832.

30. Illing PT, et al. Immune self-reactivity triggered by drug-modified HLA-peptide repertoire. Nature. 2012;486(7404):554-558.

31. Mallal S, et al. Association between presence of HLA-B ${ }^{\star} 5701$, HLA-DR7, and HLA-DQ3 and hypersensitivity to HIV-1 reverse-transcriptase inhibitor abacavir. Lancet. 2002;359(9308):727-732.

32. Norcross MA, et al. Abacavir induces loading of novel self-peptides into HLA-B*57: 01: an autoimmune model for HLA-associated drug hyper- sensitivity. AIDS. 2012;26(11):F21-F29.

33. Vivian JP, et al. Killer cell immunoglobulinlike receptor 3DL1-mediated recognition of human leukocyte antigen B. Nature. 2011;479(7373):401-405.

34. Chen $\mathrm{H}$, et al. TCR clonotypes modulate the protective effect of HLA class I molecules in HIV-1 infection. Nat Immunol. 2012;13(7):691-700.

35. [No authors listed]. 1993 revised classification system for HIV infection and expanded surveillance case definition for AIDS among adolescents and adults. MMWR Recomm Rep. 1992;41(RR-17):1-19.

36. Migueles SA, et al. The differential ability of HLA B ${ }^{*} 5701^{+}$long-term nonprogressors and progressors to restrict human immunodeficiency virus replication is not caused by loss of recognition of autologous viral gag sequences. J Virol. 2003;77(12):6889-6898.

37. Cella M, Longo A, Ferrara GB, Strominger JL, Colonna M. NK3-specific natural killer cells are selectively inhibited by Bw4-positive HLA alleles with isoleucine 80. J Exp Med. 1994;180(4):1235-1242.

38. Boudreau JE, Mulrooney TJ, Le Luduec JB, Barker E, Hsu KC. KIR3DL1 and HLA-B density and binding calibrate NK education and response to HIV. J Immunol. 2016;196(8):3398-3410.

39. Gumperz JE, et al. Conserved and variable residues within the Bw4 motif of HLA-B make separable contributions to recognition by the NKB1 killer cell-inhibitory receptor. J Immunol. 1997;158(11):5237-5241.

40. Saunders PM, et al. Killer cell immunoglobulinlike receptor 3DL1 polymorphism defines distinct hierarchies of HLA class I recognition. J Exp Med. 2016;213(5):791-807.

41. O'Connor GM, et al. Mutational and structural analysis of KIR3DL1 reveals a lineagedefining allotypic dimorphism that impacts both HLA and peptide sensitivity. J Immunol. 2014;192(6):2875-2884.

42. Norman PJ, et al. Unusual selection on the KIR3DL1/S1 natural killer cell receptor in Africans. Nat Genet. 2007;39(9):1092-1099.

43. Rojo S, Wagtmann N, Long EO. Binding of a soluble p70 killer cell inhibitory receptor to HLA-B ${ }^{*}$ 5101: requirement for all three p70 immunoglobulin domains. Eur J Immunol. 1997;27(2):568-571.

44. Khakoo SI, Geller R, Shin S, Jenkins JA, Parham P. The DO domain of KIR3D acts as a major histocompatibility complex class I binding enhancer. JExp Med. 2002;196(7):911-921.

45. Stewart-Jones GB, et al. Structures of three HIV-1 HLA-B ${ }^{*} 5703$-peptide complexes and identification of related HLAs potentially associated with long-term nonprogression. J Immunol. 2005;175(4):2459-2468.

46. Phair J, et al. Acquired immune deficiency syndrome occurring within 5 years of infection with human immunodeficiency virus type-1: the 


\section{RESEARCH ARTICLE}

Multicenter AIDS Cohort Study. J Acquir Immune Defic Syndr. 1992;5(5):490-496.

47. Vlahov D, et al. The ALIVE study, a longitudinal study of HIV-1 infection in intravenous drug users: description of methods and characteristics of participants. NIDA Res Monogr. 1991;109:75-100.

48. Emu B, et al. Phenotypic, functional, and kinetic parameters associated with apparent $\mathrm{T}$-cell control of human immunodeficiency virus replication in individuals with and without antiretroviral treatment. JVirol. 2005;79(22):14169-14178.
49. Migueles SA, et al. HIV-specific CD8 ${ }^{+} \mathrm{T}$ cell proliferation is coupled to perforin expression and is maintained in nonprogressors. Nat Immunol. 2002;3(11):1061-1068.

50 . Holcomb CL, et al. A multi-site study using high-resolution HLA genotyping by next generation sequencing. Tissue Antigens. 2011;77(3):206-217.

51. Martin MP, Carrington M. KIR locus polymorphisms: genotyping and disease association analysis. Methods Mol Biol. 2008;415:49-64.

52. Thomas R, et al. Novel KIR3DL1 alleles and their
The Journal of Clinical Investigation

expression levels on NK cells: convergent evolution of KIR3DL1 phenotype variation? J Immunol. 2008;180(10):6743-6750.

53. Clements CS, et al. The production, purification and crystallization of a soluble heterodimeric form of a highly selected T-cell receptor in its unliganded and liganded state. Acta Crystallogr $D$ Biol Crystallogr. 2002;58(Pt 12):2131-2134.

54. Barnstable CJ, et al. Production of monoclonal antibodies to group A erythrocytes, HLA and other human cell surface antigens-new tools for genetic analysis. Cell. 1978;14(1):9-20. 